\title{
MANOS INHÁBILES E HISTORIA DEL ESPAÑOL DE AMÉRICA: UN EJEMPLO DE LA NICARAGUA DIECIOCHESCA
}

\author{
UNABLE HANDS AND HISTORY OF LATIN \\ AMERICAN SPANISH: AN EXAMPLE OF 18TH. \\ CENTURY NICARAGUA
}

\author{
José Luis Ramírez Luengo \\ Universidad Autónoma de Querétaro \\ jose_ramirez14@hotmail.com
}

DOI

Resumen

Tras analizar las principales características de los textos producidos por las llamadas manos inhábiles - escriptores que, aun siendo alfabetizados, no han adquirido plena competencia en el uso de la escritura-, se edita uno de estos documentos (una oración heterodoxa nicaragüense de la primera mitad del siglo XVIII) y se lleva a cabo el análisis de los diferentes fenómenos fonéticos y fonológicos que aparecen en él. A partir de aquí, se determina que durante este periodo el español de la región presenta en tal nivel lingüístico una configuración muy similar a la que se describe actualmente en la bibliografía (Lipski, 1996), y se hace hincapié una vez más en la importancia de documentos como estos para la más correcta y completa reconstrucción histórica del español americano.

Palabras Clave: historia del español de América, Nicaragua, siglo xvin, mano inhábil, nivel fonético-fonológico

\section{Abstract}

After analysing the main features of texts which are produced by so-called unable hands - that is, writers who have not achieved complete competence in the use of writing, even if they are literate-, I edit one of these documents (a Nicaraguan unorthodox prayer from the first half of 18th. century), and I carry out the study of different phonetic and phonological phenomena which appear on it. This analysis proves that, during the 18th. century, the configuration of this linguistic level in Nicaraguan Spanish is very similar to the one that is usually described today for this regional variety (Lipski, 1996), and this conclusion allows to point out the importance of this type of documents for a correct and complete historical reconstruction of Latin American Spanish. 
KEYwORDS: history of Latin American Spanish, Nicaragua, 18th. century, unable hands, phonetic-phonological level

FECHA DE RECEPCIÓN: 30/10/2017

FECHA DE ACEPTACIÓN: 09/03/2018

\section{Manos inhábiles e historia de la lengua}

El hecho - más que evidente- de que la reconstrucción del devenir diacrónico del español de América pase necesariamente por el trabajo con documentos históricos ${ }^{1}$ ha obligado a los investigadores a meditar, antes de lanzarse a la tarea, sobre aspectos metodológicos muy diversos, entre los que sin duda destaca la relación que existe entre la lengua y su plasmación en el texto y, como clara consecuencia de lo anterior, las características que ha de poseer un corpus para que resulte útil a la hora de obtener los datos necesarios para reconstruir la situación que, en diversas épocas, presenta el español empleado en el Nuevo Mundo; ${ }^{2}$ teniendo en cuenta que la primera de las cuestiones ha sido tratada en abundantes ocasiones y desde numerosos puntos de vista - a modo de ejemplo, Pontecorvo y Blanche-Benveniste (1993)—, quizá sea interesante en esta ocasión pasar directamente al análisis del segundo de los puntos mencionados, es decir, al de los materiales que resultan especialmente valiosos para los historiadores de la lengua.

En este sentido, es verdad que cualquier documento histórico puede ser utilizado por el investigador - por cuanto ofrece

\footnotetext{
${ }^{1}$ En realidad, no siempre tan evidente para todos los investigadores, algo que obliga a Frago (1999: 8) a recordar que únicamente a partir del análisis de los testimonios escritos "puede sentarse doctrina y no a la inversa, pues la pretensión de levantar una construcción histórica sin textos de la época investigada es lo más contradictorio y banal que en nuestra humanística ciencia cabe, y lo más reñido con la filología”.

${ }^{2}$ En relación parcial con esta cuestión -y aunque no centradas específicamente en el caso americano-, son interesantes algunas de las reflexiones recogidas en Kabatek (2016).
} 
información sobre la situación lingüística de la época en que ha sido redactado-, pero no lo es menos que existen ciertos escritos que resultan mucho más interesantes que otros, pues dejan traslucir de una manera más clara la realidad lingüística que se esconde tras ellos: en efecto, si se acepta que "un texto no es un hecho aislado de comunicación, sino que la historia y la convención cultural ha ido estableciendo unos modelos" (Bustos Tovar, 2000: 69) y que, por tanto, esa tradición heredada oculta — naturalmente, de modo parcial- las características propias del autor que lo generó, no cabe duda de que para descubrir tales características resultarán especialmente valiosos aquellos escritos en los que el peso de la tradición es menor y, precisamente por eso, reflejan mejor los fenómenos que, de otro modo, quedan sepultados bajo esos modelos históricamente establecidos.

Teniendo en cuenta lo anterior, cabe preguntarse ahora quién produce los documentos que cuentan con las características que se han señalado en el párrafo anterior, y la respuesta se encuentra generalmente en aquellos hablantes poco familiarizados con la lengua escrita que tienen la destreza suficiente como para confeccionar un texto pero que por determinadas circunstancias no han adquirido el conjunto de tradiciones que establece la práctica social de la escritura, es decir, unos hablantes que - en una descripción quizá poco precisa, pero fácilmente entendible- escriben como hablan y que, al no respetar las convenciones del código escrito, permiten obtener una visión más aproximada de la situación lingüística que existe en un momento concreto; aunque son varias las denominaciones que han recibido estos escriptores en español — tales como escritores inexpertos (Blanche-Benveniste, 1998), a partir de scripteurs maladroits, o semicultos (Petrucci, 2000) - , ${ }^{3}$ en este caso se opta por

\footnotetext{
${ }^{3}$ En concreto, este autor establece una interesante diferencia entre semicultos - entendidos desde la historia de la lengua como "aquellos que, aun siendo alfabetizados, no han adquirido plena competencia en el uso de la escritura y por ello permanecen siempre ligados a la esfera de la oralidad" (Petrucci, 2000: 69) - y semialfabetizados, que en los estudios sociopaleográficos se definen como "aquellos que no abandonan las prácticas escritorias elementales, propias de quien no ha superado los niveles más primarios de la enseñanza de la escritura" (Petrucci, 2000: 69); cabe indicar que
} 
manos inhábiles, traducción del término portugués mão inábil propuesto por Marquilhas (2000: 235). ${ }^{4}$

De este modo, la cercanía a la oralidad que identifica a la documentación generada por las manos inhábiles pone claramente en evidencia su relevancia en los estudios acerca de la diacronía de las lenguas, pues permite demostrar y fechar la existencia de fenómenos de gran interés para el mejor conocimiento de su devenir histórico que en otros textos resultan difíciles de atestiguar; así las cosas, no sorprende que se hayan empleado de forma abundante en las investigaciones acerca de la historia del español, tanto de España (a manera de ejemplo, Gómez Seibane y Ramírez Luengo, 2002; Isasi Martínez y Enríquez Fernández, 2011; Sánchez-Prieto y Flores Ramírez, 2005: 24-31) como de América (entre otros, Coll, 2008; Company, 1993; Elizaincín, 1994; Ramírez Luengo y Velázquez Patiño, 2014), y de hecho son precisamente los fructíferos resultados obtenidos en estudios como los citados los que avalan la importancia de estos materiales para obtener una visión más profunda y detallada -y por tanto más realista- de las características que presenta el español en una etapa específica de su historia.

\section{El corpus: una versión nicaragüense de la Oración del Santo Sepulcro}

Partiendo, pues, de todo lo indicado hasta el momento, el presente trabajo persigue ofrecer una prueba más del evidente interés que poseen los escritos generados por las manos inhábiles a la hora de llevar a cabo la reconstrucción histórica del español de América; con este propósito, se pretende realizar la edición y

\footnotetext{
- aunque no necesariamente- en numerosas ocasiones ambas categorías confluyen en un mismo escriptor.

${ }^{4}$ Sus textos, por tanto, son reflejo de lo que se ha denominado en múltiples ocasiones 'competencia escrita de impronta oral'; véanse a este respecto los trabajos de Oesterreicher $(1992,1996)$ para la descripción de las características de tales documentos y su coincidencia - frecuente, pero no absoluta - con los productos escritos de las manos inhábiles.
} 
el estudio de un texto perteneciente a esta categoría que - como se intentará demostrar más adelante - resulta de notable importancia para describir la variedad del español de Nicaragua en el siglo xVIII, y muy especialmente la configuración fónica que esta presenta ya en la primera mitad del Siglo Ilustrado.

Por lo que se refiere al documento en sí, se trata de una oración heterodoxa muy breve —unas 500 palabras aproximadamente- que José de Vidaurre, comisario del Santo Oficio de la Inquisición en León, remite desde esta población nicaragüense al tribunal de la Ciudad de México, donde hoy se conserva entre los fondos inquisitoriales del Archivo General de la Nación (AGN). ${ }^{5}$ Dado que el texto se acompaña de un memorial - fechado en la misma León el día tres de enero de 1737- en el que el propio Vidaurre indica el descubrimiento de dos oraciones "entre la gente bulgar que paresen superstisiosas que ê recojido y remito (...) para $\mathrm{q}<\mathrm{ue}>$ sobre el asunto me mande quanto deuo executar", ${ }^{6}$ parece posible localizar el escrito estudiado (que carece de data tópica y crónica) en esta ciudad o sus alrededores en algún momento de la primera mitad de la década mencionada; así mismo, es también interesante la referencia del denunciante sobre la "gente bulgar" de quien ha tomado la oración, pues se puede interpretar como un indicio del nivel sociocultural del autor de la misma y constituye, en unión a los datos anteriores, el marco de referencia que, de acuerdo con los principios de la dialectología histórica, permite interpretar y valorar de manera más precisa los fenómenos lingüísticos que se extraerán del análisis.

En cuanto a su contenido, se trata, según se ha dicho ya, de una versión de la denominada Oración del Santo Sepulcro, presente en España ya en el mismo siglo xvi y popular en la Nueva España desde entonces y hasta al menos el siglo xviII (Campos Moreno, 2006: 289, 292); ${ }^{7}$ en concreto, el texto aquí editado na-

\footnotetext{
${ }^{5}$ En concreto, bajo la signatura AGN (México), Inquisición (61), vol. 854, p. 359r.-v. ${ }^{6}$ El memorial acompaña al texto de la oración que se va a editar, y se encuentra en la página 358r.-v. del legajo mencionado en el nota anterior; específicamente, la cita reproducida aparece en la página $358 \mathrm{v}$.

${ }^{7}$ En realidad, hasta el momento actual, pues la propia autora narra en su trabajo el
} 
rra la aparición de Cristo a santa Brígida y santa Isabel de Hungría para detallarles los pormenores de la pasión e indicarles las heridas, los golpes que recibió e incluso las lágrimas y gotas de sangre que derramó, a partir de lo cual -y siempre y cuando el lector cumpla una serie de requisitos- se le prometen ciertos beneficios, tales como conocer el día de su muerte, ser acompañado por Cristo y la Virgen en ese trance o quedar protegido frente a determinados accidentes mortales imprevistos, así como durante el parto en el caso de las mujeres. Salta a la vista, por tanto, lo alejado de la ortodoxia católica ${ }^{8}$ que se encuentra un documento como este y las pocas posibilidades que existen en principio de que logre pasar a la posterioridad; sin embargo, es precisamente este carácter heterodoxo lo que en este caso explica su conservación, pues tal circunstancia le permite trascender su propia finalidad utilitaria y transformarse, por mor del celo inquisitorial, en un pieza más de la copiosa documentación que genera la minuciosa - y asfixiante- burocracia del Santo Oficio. ${ }^{9}$

Por último, cabe mencionar que la edición se ha realizado siguiendo los criterios de la Red Internacional CHARTA (CHARTA, 2015) en su triple presentación - esto es, una transcripción paleográfica, una presentación crítica y una versión fac-

descubrimiento de un ejemplar impreso de la Oración en la puerta de una iglesia de la capital mexicana en 1997 (Campos Moreno, 2006: 296); a este respecto, resulta especialmente interesante el hecho de que la versión nicaragüense que aquí se presenta se corresponda de forma casi literal con la mencionada por la profesora de la Universidad Nacional Autónoma de México, tanto en su contenido como en lo que se refiere a algunas de las recompensas que, por intercesión celestial, recibirá el devoto. ${ }^{8} \mathrm{Si}$ bien profundamente enraizado en la religiosidad popular, como demuestra su transmisión más o menos clandestina desde Nicaragua hasta México, y desde el siglo XVIII hasta finales del siglo xx.

${ }^{9}$ Esta idea no es aplicable únicamente a los dominios del imperio español: como señala para el caso de Portugal Marquilhas, "o aparelho judicial do Santo Ofício foi responsabilizado pela conservação de provas da competência gráfica de personagens que não tinham notoriedade social ou cultural" (2000: 229); de ahí el interés que tiene la documentación generada por esta institución y su empleo para la confección de antologías, tanto de carácter lingüístico (por ejemplo, Company, 1994; o Ramírez Luengo, 2017a, 2017b) como relacionadas con la literatura y las tradiciones populares (Flores y Masera, 2010). 
similar-, a lo que se añade un estudio del texto dividido en dos partes diferenciadas: por un lado, se llevará a cabo un análisis de las características - tanto formales como escriturarias- que permiten calificarlo desde tales puntos de vista como producto de una mano inhábil (Marquilhas, 2000: 238-241; Sánchez-Prieto y Flores Ramírez, 2005: 25-26); por otro, se desarrollará un estudio de todas las características del nivel fonético-fonológico que se evidencian en el documento con el propósito de describir la configuración fónica que presenta en esta época el español nicaragüense. ${ }^{10}$ Tras estos dos puntos, el trabajo se concluye con un último apartado en el que se determinará cuál es el grado de semejanza fónica que se descubre entre la situación de la Nicaragua del Setecientos y la que se describe actualmente para este país (Lipski, 1996: 311-313; Rosales Solís, 2010), así como con una valoración, a partir de este caso concreto, de las ventajas que presenta una documentación como la aquí analizada para los estudios sobre la historia del español americano.

\section{El texto estudiado, I: características formales y escriturarias de los escritos inhábiles}

Por lo que se refiere a esta primera cuestión, una observación superficial del documento estudiado permite identificar fácilmente su carácter de texto producido por una mano inhábil, pues en él se descubren todas las características formales que

\footnotetext{
${ }^{10}$ Para esta cuestión se ha optado por utilizar la metodología que habitualmente se emplea en los estudios sobre la evolución fónica del español (Frago, 1999; 2002: 409495), basada en la detección de aquellas grafías del texto que suponen una desviación respecto a las tendencias gráficas del momento (Ramírez Luengo, 2012a: 168) y en su interpretación como reflejo de determinados fenómenos fónicos que caracterizan el habla del escriptor pero no tienen cabida en ellas; por supuesto, si bien es verdad que, como señalan Sánchez-Prieto y Flores Ramírez, "la valoración fonética de cualquier testimonio presenta serias dificultades, pues mientras que la grafía es patente, a una misma secuencia gráfica pueden corresponder diversos valores fonéticos", y de ahí que "cualquier intento de valoración fonética sea tentativo" (2005: 27), no lo es menos que tal metodología ha sido profusamente utilizada por los investigadores interesados en esta cuestión y ha aportado datos de indudable valor para el mejor conocimiento de la fonología histórica del español.
} 
Marquilhas (2000: 238) considera propias de tales escritos: en efecto, no es difícil observar en el facsímil cuestiones como la incapacidad de alinear perfectamente las palabras siguiendo la horizontalidad - presente en todo el texto, pero especialmente en lugares como las líneas $12,13,17,18$ y 19 del folio recto- $-{ }^{11}$ o la tendencia a conferir a las letras una apariencia desencuadrada y al empleo del módulo grande (a manera de ejemplo, h 1r. 24, 25), a lo que se suma el trazado muy inseguro y no continuado de la escritura, detectable en Attado ala (h 1r. 21), o la ausencia de elementos de ligación entre las grafías que se descubre, por ejemplo, en Jesu o abe maria (h 1r. 2, 29), ${ }^{12}$ todo lo cual produce cierta rigidez y falta de ligereza en el conjunto que diferencia claramente un documento como este de aquellos que son generados por personas más entrenadas o habituadas en la práctica de la escritura. ${ }^{13}$

Junto a lo anterior, y más allá de lo formal, el texto ofrece una serie de rasgos escriturarios que también resultan recurrentes en las piezas producidas por las manos inhábiles y que, por tanto, también pueden ser empleados para su caracterización: a este respecto, tanto Marquilhas (2000: 238-239) como SánchezPrieto y Flores Ramírez (2005: 25-26) indican como propias de la impericia gráfica cuestiones presentes en la Oración como

\footnotetext{
${ }^{11}$ En relación con la disposición de la escritura en el soporte, otra característica muy propia de los escritos inhábiles es la inexistencia de márgenes, algo que también se aprecia claramente en este, por ejemplo a lo largo de toda la hoja 1r., tanto en el lateral derecho como en la parte inferior.

${ }^{12}$ Marquilhas (2000: 238) señala también la ausencia casi total de abreviaturas como un fenómeno propio de los textos inhábiles, pero no es esta la situación del documento aquí trascrito, que utiliza algunas como $S a n<t i d a>, p<o r>$ o $q<u e>$ (h 1r. 4, 9; $\mathrm{h} 1 \mathrm{v} .12$ ), a veces incluso formales - con presencia de letras voladas- pero sin valor abreviativo, tal y como se aprecia en $S S<u>$ (h 1r. 4); de todas formas, esta misma autora indica que, por lo que se refiere a sus documentos, "a presença de abreviaturas não é característica rara” (Marquilhas, 2000: 238), algo que también descubren en su documentación hispánica Sánchez Prieto y Flores Ramírez (2005: 26).

${ }^{13}$ Estos fenómenos no son propios únicamente de los siglos XVI o XVII, sino que todos ellos se pueden ver claramente ejemplificados también en los bonos de la Vizcaya decimonónica que analizan en su estudio Isasi Martínez y Enríquez Fernández (2011), así como en los memoriales del centro peninsular de esta época que describe Almeida Cabrejas (2014: 169-177).
} 
el empleo indiscriminado de mayúsculas y minúsculas —en el caso aquí analizado, sepulCro, LaCual, Deungria, enora Sion, HiJas (h 1r. 4, 6, 7) - o la segmentación gráfica no acorde con lo que dicta la tradición (sol dados, Mediero, h 1r. 11, 20; sumuerte, Li bre de to dos Peli Gros, h 1v. 4, 6), a lo que se pueden sumar otros fenómenos como la aparición de usos gráficos que rompen las tendencias de la época ( $m y L$, dyos, h 1r. 21, 24; $m y$, h 1v. 3), la ausencia de tildación - que en este texto es absoluta- o la puntuación irregular, sea en forma de sobrepuntuación o sea en forma de ausencia total, situación que muestra este documento nicaragüense.

De este modo, no cabe duda de que existe una serie de fenómenos formales y escriturarios que permiten definir el texto aquí estudiado como producto de una mano inhábil; teniendo esto en cuenta, parece el momento de analizar qué rasgos caracterizan, también desde el punto de vista lingüístico, a estos documentos y, en consecuencia, llevar a cabo una valoración de su importancia para los estudios que pretenden reconstruir la evolución diacrónica del español empleado en el Nuevo Mundo.

\section{El texto estudiado, II: las manos inhábiles y el español nicaragüense del siglo XVIII}

Por otro lado, el desconocimiento que muestran las manos inhábiles de las convenciones sociales que rigen el acto de la escritura determina que en sus textos aparezcan algunos fenómenos lingüísticos que, en el caso de individuos con mayor habilidad o familiaridad con esta práctica, quedan ocultos por la tradición que rige sus usos; no sorprende, por tanto, que en tales escritos sea relativamente frecuente la plasmación gráfica de rasgos de la pronunciación que habitualmente no se reflejan en los usos gráficos aceptados o la presentación de fenómenos morfosintácticos que son propios de la oralidad y que representan estrategias características de esta - tales como focalizaciones, anacolutos o sobreentendidos-, así como, desde el punto de vista del vocabulario, la presencia de cualquier tipo de unidades léxicas, entre 
ellas las más populares o las diatópicamente marcadas que, en muchas ocasiones, por quedar fuera del discurso convencionalmente aceptado, no tienen cabida en otro tipo de documentos.

Así las cosas, salta a la vista que esta documentación resulta de interés para el estudio de cualquiera de los niveles que componen el sistema lingüístico, pero no cabe duda de que su importancia es todavía mayor para el caso de lo fónico, habida cuenta de que, como bien señalan Isasi Martínez y Enríquez Fernández, en estos escritos "son visibles rasgos más cercanos a la oralidad y también, en consecuencia, a los rasgos idiolectales de los redactores" (2011: 422); haciendo propias, por tanto, las palabras de los investigadores vascos y aplicándolas a este caso específico, se puede concluir que el texto que se está considerando en estas páginas constituye, por la información lingüística que aporta, un documento de primera importancia para el estudio de la situación que presenta el español nicaragüense del siglo XVIII, y más específicamente para establecer su configuración fónica en la época mencionada, tarea a la que se dedicarán los párrafos siguientes.

De este modo, la presencia de las amalgamas destos, desta (h 1r. 28; h 1v. 2) parece evidenciar - siempre y cuando no sea una mera cuestión gráfica, habida cuenta de su larga tradición en la escritura- "la connivencia entre lo fónico y lo grafémico manifestada a propósito de pronunciaciones bastante corrientes" (Frago, 1999: 209-210) en español, en esta ocasión en forma de simplificaciones de vocales en contacto que, si bien presentan carácter general y por tanto escaso interés dialectal, sirven en todo caso para demostrar la aparición de los "rasgos cercanos a la oralidad” señalados por Isasi Martínez y Enríquez Fernández (2011: 422) y avalan, al mismo tiempo, la pertinencia del método de análisis que se está utilizando en este estudio.

Más allá de esto, los fenómenos vocálicos que se descubren en el texto son tres: por un lado, la vacilación en las vocales átonas (padisi, h 1r. 8; confision, h 1v. 11), frecuente todavía en América durante el Setecientos (Ramírez Luengo, 2007: 40) y que, a la luz de estos ejemplos y de los de Ramírez Luengo (2012b: 295), constituye un rasgo presente en todos los grupos 
sociales de la Nicaragua de la época; ${ }^{14}$ por otro, la diptongación de los hiatos que parece ejemplificar el caso de Guaquín 'Joaquín' (h 1v. 22), localizable en la práctica totalidad del Nuevo Mundo desde antiguo (Ramírez Luengo, 2007: 41); por último, las monoptongaciones registradas en pusttose 'puéstose' (h $1 \mathrm{r}$. 6) o fi 'fui' (h 1r. 19), que pueden reflejar la pronunciación del autor - de hecho, como inseguridades vocálicas o vulgarismos interpreta Frago (1999: 250, 273) apariciones americanas semejantes-, pero que también es posible considerar como simples muestras de la impericia del autor a la hora de representar los diptongos y, por tanto, meros errores gráficos carentes de cualquier trascendencia fónica. ${ }^{15}$

Frente a la escasez de fenómenos vocálicos que se descubre en el texto, el análisis del consonantismo ofrece datos de notable trascendencia para el objetivo último de describir la configuración fónica del español nicaragüense del siglo XVIII: en este sentido, no cabe duda de que una de las cuestiones de mayor relevancia, tanto por su importancia dialectal como por su profusa aparición en la Oración, es la abundante confusión de grafías que denota el seseo, que se descubre en voces como, entre otras, orasion, aparesio, siento, tresientas, dies, sinco, beses, gotaz o pazion (h 1r. 6, 7, 11, 13, 15, 20, 23, 26, 28); por supuesto, es evidente que la rápida generalización de tal pronunciación por América (Ramírez Luengo, 2007: 33-34) auguraba su aparición en un texto del Setecientos como este, de manera que, si bien no

\footnotetext{
${ }^{14}$ No parece casual que ambas ocurrencias muestren el fenómeno precisamente en la pareja palatal /e/-/i/, pues una situación semejante — con el mantenimiento de la vacilación exclusivamente en este par vocálico- presenta, por ejemplo, el español salvadoreño del momento (Ramírez Luengo, en prensa).

${ }^{15}$ A estos dos ejemplos quizá se pueda sumar también el caso de deseas (h 1r. 8), si bien resulta dudoso y puede tener una doble interpretación: así, si se considera una cacografía por 'deseáis', entonces indudablemente se debe interpretar como una muestra más de los problemas - puramente gráficos o de pronunciación- que afectan a los diptongos en el texto; si, por el contrario, se trata de un error por 'desean', en ese caso la cacografía parece apuntar hacia el debilitamiento de las consonantes implosivas /-s/ y /-n/, fenómenos que forman parte del consonantismo débil que hoy caracteriza al español de Nicaragua (Lipski, 1996: 311-313; Rosales Solís, 2010: 151) y que, como se verá posteriormente, se hacen ya presentes de forma inequívoca en el documento estudiado.
} 
sorprende la constatación de la naturaleza seseante del autor del escrito, en todo caso es interesante señalarla, no solo porque demuestra la antigüedad del fenómeno actual en la zona (Rosales Solís, 2010: 143), sino también porque permite, en unión con los datos de Ramírez Luengo (2012b: 295), atestiguar la generalidad del seseo en todos los niveles sociales de la Nicaragua del siglo XVIII.

Por su parte, algunas grafías del corpus como sullo, llo, rallos o alludara 'ayudará' (h 1r. 5, 31; h 1v. 12, 17) muestran bien a las claras la presencia indudable de yeísmo en el habla del autor del texto, lo que resulta coincidente con el español empleado en la zona en el momento actual (Lipski, 1996: 311; Rosales Solís, 2010: 146) pero contrasta con la situación descrita para los estratos cultos de esta misma región en el siglo XVIII, donde parece ser desconocido (Ramírez Luengo, 2012b: 296), de manera que es necesario plantearse a la luz de estos datos si existe en la época una distribución de este fenómeno de corte sociolingüístico y, en caso de que así sea, en qué momento se modifica tal situación a favor de la generalización yeísta que identifica hoy al español hablado en Nicaragua.

Más allá del seseo y del yeísmo, la aspiración de la /s-/ implosiva constituye también al día de hoy uno de los fenómenos caracterizadores de la variedad nicaragüense del español (Lipski, 1996: 312-313; Rosales Solís, 2010: 144-146); pues bien, una vez más los datos del corpus analizado, con cacografías que denotan su presencia - tales como la puñadas (h 1r. 13), destos paso (h 1r. 28) o dieros 'dieron' (h 1r. 17) —, ${ }^{16}$ se suman a las atestiguaciones que al respecto se aportan en Ramírez Luengo (2012b: 295-296), y permiten, de este modo, esbozar una situación semejante a la actual en este punto concreto del sistema ya

${ }^{16}$ En principio también podría considerarse un caso de aspiración la locución preposicional en cas de Anas 'en casa de Anás' (h 1r. 16) si se considera ese cas como ultracorrección gráfica de $c a$, forma simplificada de casa muy frecuente en las hablas populares (Menéndez Pidal, 1962: 79); sin embargo, el hecho de que Autoridades (RAE, 1979: s.v. cas) recoja la forma cas con este mismo valor obliga a ser precavidos a la hora de interpretar este supuesto ejemplo de aspiración. 
durante las primeras décadas del siglo XVIII. Al mismo tiempo, es importante volver al dieros 'dieron' ya mencionado porque, junto a la aspiración de la /-s/, demuestra también la existencia de cierto debilitamiento de la /-n/ implosiva que produce ultracorrecciones como la anterior, cuestión que se ve corroborada por la aparición de un resare 'rezaren' (h 1r. 27) y varios ejemplos de diero 'dieron' en el texto (h 1r. 20, 23) ${ }^{17}$ que quizá sean reflejo, en realidad, del empleo del alófono velarizado en tal posición y de la posterior nasalización de la vocal, una vez más en coincidencia con la situación que, al respecto, describen para el momento actual tanto Lipski (1996: 312) como Rosales Solís (2010: 149-150).

Por otro lado, la presencia de un consonantismo débil que parecen mostrar tanto la aspiración de la /-s/ implosiva como la velarización de la /-n/ en tal posición se ve reforzada también por la elisión de otros fonemas en el texto, tales como la /d/ -que se descubre de forma clara en humilda (h 1v. 15), así como en un santidá abreviado (h 1r. 4) ${ }^{18}$ y quizá en abe (h 1v. 20), imperativo voseante del verbo haber - o la $/ \mathrm{r} /$, especialmente en posición final, tal y como refleja un seño 'señor' reiterado a lo largo de todo el texto (h 1r. 2, 5, 7, 13, 22, 26; h 1v. 16), pero quizá también en grupo consonántico, si es que el único ejemplo al respecto, nuesta 'nuestra' (h 1v. 17), no constituye un mero lapsus cálami. ${ }^{19}$ Así pues, parece posible concluir que la existencia de los fenómenos que se acaban de describir, presentes actualmente con mayor o menor frecuencia en la región (Lipski,

\footnotetext{
${ }^{17}$ Así como por el deseas citado en la nota 15, siempre y cuando se considere, naturalmente, un error gráfico por la tercera persona del plural desean.

${ }^{18}$ Cabe indicar que las letras voladas que marcan la abreviatura son $<$ da $>$, lo que parece demostrar la elisión de la /d/ final, de cuya existencia ni siquiera es consciente el escriptor a la hora de abreviar el término.

${ }^{19}$ Se debe mencionar la aparición también en el corpus de algunas confusiones entre $<\mathrm{r}>\mathrm{y}<\mathrm{rr}>$ en posición interior de palabra (tiera, arenpugones, derame; h 1r. 18, 23, $25,31)$, si bien es probable que se trate de meros errores gráficos, semejantes a los que Frago (2007: 155-156) registra en múltiples zonas de la América colonial; quizá como tal deba interpretarse, así mismo, la adición de una $<\mathrm{l}>$ al posesivo mi, que se puede ver favorecida por la aparición del numeral mil en una posición muy cercana: Que daron en MiL Cuerpo myL eridas (h 1r. 22-23).
} 
1996: 311; Rosales Solís, 2010: 150), se puede retrotraer al menos hasta la Centuria Ilustrada, y que en este siglo constituye una característica compartida por los distintos grupos de la sociedad colonial nicaragüense, dado que ejemplos semejantes a estos se descubren en los documentos cultos analizados en Ramírez Luengo (2012b: 295).

Finalmente, es importante señalar unas últimas cuestiones acerca de la configuración fónica del español nicaragüense de la época que también es posible deducir del texto, si bien su atestiguación es mucho más escasa: más allá de la muy dudosa despalatalización que quizá muestre aconpanarlo (h $1 \mathrm{v}$. 3), es posible que grafías como digo 'dijo', arenpugones 'arrempujones' o Guaquin 'Joaquín' (h 1r. 7, 23; h 1v. 22) constituyan —en caso de no ser meros lapsus cálami, sin trascendencia fónica algunaejemplos del empleo del alófono laríngeo o débil [h] del fonema /x/, tal como ocurre actualmente en la región (Lipski, 1996: 311; Rosales Solís, 2010: 148-149); por último, cabe señalar que el único ejemplo de grupos cultos que se utiliza en el corpus, coluna 'columna' (h 1r. 21) opta por la solución simplificada, algo que contrasta con el empleo de los documentos dieciochescos de nivel socioeducacional alto, en los que la preferencia por la reposición de la oclusiva implosiva es claramente mayoritaria (Ramírez Luengo, 2012b: 297), lo que parece demostrar — por supuesto, desde la provisionalidad que conlleva basarse en ejemplos tan escasos- la imposición, ya en esta época, de una distribución sociolingüística de este fenómeno semejante a la que se descubre hoy en día en todo el mundo hispánico.

Así pues, la comparación de los documentos cultos de Ramírez Luengo (2012b) y de la Oración de la mano inhábil objeto de este trabajo permite establecer ya una serie de diferencias en cuanto a los fenómenos fónicos que se reflejan en ambos corpus, tal y como se registra en el cuadro siguiente:

\begin{tabular}{|c|c|c|}
\hline Fenómeno & Nivel Bajo & Nivel Alto \\
\hline Vacilaciones en vocalismo átono & $\mathrm{X}$ & $\mathrm{X}$ \\
\hline Diptongación de los hiatos & $\mathrm{X}$ & \\
\hline Problemas con diptongos & $\mathrm{X}$ & \\
\hline
\end{tabular}




\begin{tabular}{|c|c|c|}
\hline Fenómeno & Nivel bajo & Nivel alto \\
\hline Seseo & $\mathrm{X}$ & $\mathrm{X}$ \\
\hline Yeísmo & $\mathrm{X}$ & $\mathrm{X}$ \\
\hline Aspiración de /-s/ implosiva & $\mathrm{X}$ & \\
\hline $\begin{array}{c}\text { Velarización/elisión de /-n/ } \\
\text { implosiva }\end{array}$ & $\mathrm{X}$ & $\mathrm{X}$ \\
\hline Debilitamiento/caída de/d/ & $\mathrm{X}$ & $\mathrm{X}$ \\
\hline Debilitamiento/caída de/r/ & $\mathrm{X}$ & \\
\hline Empleo de alófono [h] de /x/ & $\mathrm{X}$ & \\
\hline $\begin{array}{c}\text { Simplificación de grupos cultos } \\
\text { consonánticos }\end{array}$ & $\mathrm{X}$ & \\
\hline
\end{tabular}

Cuadro 1. Fenómenos fónicos en el español nicaragüense del siglo XVIII

Considerando, pues, que tales divergencias responden a diferencias que están determinadas desde el punto de vista diastrático, parece posible concluir que, desde el punto de vista fónico, el español utilizado en la Nicaragua del Setecientos se caracteriza por contar con una serie de fenómenos comunes en concreto, las vacilaciones en el vocalismo átono, el seseo y el consonantismo débil en forma de aspiración de la /-s/ implosiva y ciertas elisiones consonánticas- a los que se añaden otros que sirven para establecer cierto contraste entre los dos niveles socioeducacionales considerados, entre los que sin duda destacan la velarización y/o elisión de la /-n/ implosiva y el yeísmo, que claramente aparecen en los niveles populares y, sin embargo, parecen ser desconocidos en el habla que utilizan las clases altas de la región. ${ }^{20}$

\footnotetext{
${ }^{20}$ Es necesario, con todo, tener en cuenta, en un análisis como el presente, "hasta qué punto el distinto conocimiento de las normas gráficas de la época por parte de los escribientes - inherente al hecho de comparar individuos de diferente nivel educacional- no conlleva un grado dispar de traslación de la realidad fónica al texto y, por tanto, una cierta distorsión en los datos que se pueden extraer de tales textos" (Ramírez Luengo, 2015: 119), algo que sin duda dificulta en parte la valoración sociolingüística de los resultados que se obtienen gracias a esta metodología.
} 


\section{A modo de conclusión}

De este modo, el análisis llevado a cabo hasta este momento permite extraer una serie de conclusiones de notable interés a la hora de establecer de una forma más precisa la situación que, desde el punto de vista fónico, presenta el español de Nicaragua durante el segundo tercio del siglo XVIII.

A este respecto, el análisis de las grafías del texto que se salen de las tendencias de escritura propias de la época permite evidenciar la presencia, en el habla de los grupos populares de la región, de una serie de fenómenos presentes de manera más o menos general en el español del siglo xviII como las vacilaciones en el vocalismo átono o la simplificación de los grupos cultos consonánticos, a los que se suman otros diatópicamente más restringidos como el seseo, el yeísmo, la velarización de la /-n/ implosiva o el empleo del alófono [h], así como la imposición de un consonantismo débil que se refleja en la aspiración de la /s-/ implosiva y el debilitamiento y/o pérdida de ciertos fonemas como la /d/ o la /r/; se trata, por tanto, de una configuración fónica muy semejante a la que los estudiosos describen para el español nicaragüense de hoy (Lipski, 1996: 311-313; Rosales Solís, 2010), lo que demuestra que, desde este punto de vista, la situación que existe actualmente se puede retrotraer al menos hasta los primeros años de la Centuria Ilustrada.

Sin embargo, quizá las conclusiones más interesantes que se puedan extraer de un trabajo necesariamente limitado como este sean las que guardan relación con el interés que, desde un punto de vista metodológico, presentan los textos de las manos inhábiles para el estudio de la historia del español americano: así, un análisis como el que se ha esbozado a lo largo de estas páginas pone de manifiesto la importancia fundamental que adquieren tales textos para la más correcta reconstrucción de la situación lingüística que presenta una determinada variedad diatópica en un momento del pasado, ${ }^{21}$ y esto no solo porque

\footnotetext{
${ }^{21}$ Especialmente fónica, como se ha visto en estas páginas, pero no solo, pues escritos como la Oración permiten extraer también relevantes informaciones sobre la morfosintaxis o el léxico del español nicaragüense en el Siglo Ilustrado: a manera de
} 
documentan las características del habla de ciertos grupos sociales que de otro modo muy escasamente hacen acto de presencia en los corpus históricos, sino también porque al mismo tiempo permiten, por medio de su comparación con los escritos de los niveles más cultos de la sociedad, ${ }^{22}$ aportar a la investigación una perspectiva de carácter sociolingüístico que enriquece en mucho las conclusiones a las que puede llegar el estudioso y que, por tanto, facilita una visión más completa $-\mathrm{y}$ por tanto más acertada- del español empleado en el Nuevo Mundo durante un periodo concreto de su devenir histórico.

\section{Bibliografía}

Almeida Cabrejas, Belén (2014), "Scriptores con bajo y medio nivel socioeducacional en documentos del siglo xix del Archivo Municipal de Alcalá de Henares: acercamiento a sus usos gráficos", en R. Díaz Moreno y B. Almeida Cabrejas (eds.), Estudios sobre la historia de los usos gráficos en español, Lugo, Axac, pp. 167-210.

Blanche-Benveniste, Claire (1998), "Las unidades de lo escrito y lo oral”, en Estudios lingüísticos sobre la relación entre oralidad y escritura, Barcelona, Gedisa, pp. 65-104.

Bustos Tovar, José Jesús de (2000), “Texto, discurso e historia de la lengua", Revista de Investigación Lingüística, 2/3, pp. 67-94.

Campos Moreno, Araceli (2006), “La devoción de la Oración

ejemplo, véase la tendencia popular a modificar la terminación de algunas voces para que presenten la marca morfológica de su género (efigia, h 1v. 8), el mantenimiento del voseo reverencial (Abe 'habed', h 1v. 20) o el uso del futuro de subjuntivo (resare, h 1r. 27; estubiere, h 1v. 13-14); desde el punto de vista léxico, el empleo de voces (actualmente) vulgares o desusadas como puñadas, arrempujones y horas menguadas (h 1r. 13, 23; h 1v. 13).

${ }^{22}$ De ahí la importancia, tal y como se señalaba en Ramírez Luengo, "de que aparezcan en las antologías textos que representen todos los niveles sociales que componen la sincronía/diatopía que se pretende reflejar con sus materiales", pues solo así parece posible evitar "una visión sesgada de la realidad lingüística de una época" (2012c: 300). 
del Santo Sepulcro y la escritura mágica”, en P. M. Cátedra García et al. (eds.), La literatura popular impresa en España y en la América colonial: formas y temas, géneros, funciones, difusión, historia y teoría, Salamanca, Seminario de Estudios Medievales y Renacentistas, pp. 289-298.

CHARTA (2015), Criterios de edición de documentos hispánicos (orígenes-siglo XIX), en línea: <http://files.redchartal. webnode.es/200000023de670df5d6/Criterios\% 20CHARTA\%2011abr2013.pdf> [Consulta: 10/08/2015].

Coll, Magdalena (2008), "Doña Teresa de Aguilera y Roche: una mujer en la Inquisición en Nuevo México, una voz en la historia del español del sudoeste de Estados Unidos”, en A. Balestra, G. Martínez y M. I. Moyna (eds.), Recovering the U.S. Hispanic Linguistic heritage: sociolinguistic approaches to Spanish in the United States, Houston, Arte Público Press, pp. 89-108.

Company Company, Concepción (1993), "Fonética novohispana a fines del siglo XVII", Anuario de Letras, XXXI, pp. 557-575.

Company Company, Concepción (1994), Documentos lingüísticos de la Nueva España. Altiplano Central, México, Universidad Nacional Autónoma de México.

Elizaincín, Adolfo (1994), "Fructuoso Rivera como informante calificado para la historia del español en el Uruguay", en M. B. Fontanella de Weinberg (comp.), El español del Nuevo Mundo. Estudios sobre historia lingüística hispanoamericana, Washington, Organización de Estados Americanos, pp. 61-72.

Flores, Enrique y Mariana Masera (coords.) (2010), Relatos populares de la Inquisición novohispana, Madrid, Consejo Superior de Investigaciones Científicas / Universidad Nacional Autónoma de México.

Frago, Juan Antonio (1999), Historia del español de América. Textos y contextos, Madrid, Gredos.

Frago, Juan Antonio (2002), Textos y normas. Comentarios lingüísticos, Madrid, Gredos.

Frago, Juan Antonio (2007), “Tradición e innovación en el es- 
pañol americano de la Independencia”, Romance Philology, 61/2, pp. 147-191.

Gómez Seibane, Sara y José Luis Ramírez Luengo (2002), "Notas sobre la lengua de un guipuzcoano emigrado a Indias en el siglo XVIII", Estudios Lingüísticos de la Universidad de Alicante, 16, pp. 331-350.

Isasi Martínez, Carmen y Javier Enríquez Fernández (2011), "El castellano en documentos del siglo XIX: testimonios de manos inhábiles", Moenia, 17, pp. 421-434.

KabATEK, Johannes (2016), Lingüística de corpus y lingüística histórica iberorrománica, Berlín, De Gruyter.

Lipski, John M. (1996), El español de América, Madrid, Cátedra. Marquilhas, Rita (2000), A Faculdade das Letras. Leitura e escrita em Portugal no séc. XVII, Lisboa, Imprensa Nacional-Casa da Moeda.

Menéndez Pidal, Ramón (1962), Manual de gramática histórica, Madrid, Espasa-Calpe.

Oesterreicher, Wulf (1992), "El español en textos escritos por semicultos: competencia escrita de impronta oral en la historiografía indicana”, en J. Lüdtke (coord.), El español de América en el siglo XVI, Madrid / Fráncfort, Iberoamericana / Vervuert, pp. 155-190.

Oesterreicher, Wulf (1996), "Lo hablado en lo escrito: reflexiones metodológicas y aproximación a una tipología”, en T. Kotschi, W. Oesterreicher y K. Zimmermann (coords.), El español hablado y la cultural oral en España e Hispanoamérica, Madrid / Fráncfort, Iberoamericana / Vervuert, pp. 317-340.

Petrucci, Armando (2000), "Escrituras marginales y escribientes subalternos", SIGNO. Revista de Historia de la Cultura Escrita, 7, pp. 67-75.

Pontecorvo, Clotilde y Claire Blanche-Benveniste (1993), Proceedings of the Workshop on Orality versus Literacy: Concepts, Methods and Data, Estrasburgo, European Science Foundation.

Ramírez Luengo, José Luis (2007), Breve historia del español de América, Madrid, ArcoLibros. 
Ramírez Luengo, José Luis (2012a), “Notas sobre las tendencias gráficas del español colombiano en la época de las Independencias (1830)", en J. L. Ramírez Luengo (coord.), Por sendas ignoradas. Estudios sobre el español del siglo XIX, Lugo, Axac, pp. 167-182.

RAmírez Luengo, José Luis (2012b), “Una aportación a la historia de la lengua española en Nicaragua: algunos datos sobre el siglo XVIII", en Ma . T. García Godoy (ed.), El español del siglo XVIII. Cambios diacrónicos en el primer español moderno, Berna / Bruselas / Nueva York, Peter Lang, pp. 293-312.

Ramírez Luengo, José Luis (2012c), “Algunas cuestiones teóricas acerca de la edición de documentos lingüísticos americanos", en Ma J. Torrens Álvarez y P. Sánchez-Prieto (eds.), Nuevas perspectivas para la edición y el estudio de documentos lingüísticos antiguos, Berna / Bruselas / Nueva York, Peter Lang, pp. 301-310.

Ramírez Luengo, José Luis (2015), “La configuración fónica del español de la Bolivia andina en la primera mitad del siglo XIX: notas sociolingüísticas", Études Romanes de Brno, 36/2, pp. 111-123.

Ramírez Luengo, José Luis (2017a), Textos para la historia del español XI. Honduras y El Salvador, Alcalá de Henares, Universidad de Alcalá.

Ramírez Luengo, José Luis (2017b), Documentos lingüísticos de la Guatemala dieciochesca (1690-1810), Lugo, Axac.

RAmírez Luengo, José Luis (en prensa), "La configuración fónica del español salvadoreño en la época colonial (16501803)", Boletín de la Real Academia Española.

Ramírez Luengo, José Luis y Eduardo P. Velázquez Patiño (2014), "El español de los bilingües altoperuanos en la primera mitad del siglo xix: rasgos fónicos”, en J. L. Ramírez Luengo y E. P. Velásquez Upegui (coords.), La historia del español hoy. Estudios y perspectivas, Lugo, Axac, pp. 33-54. Real Academia Española (1979 [1726-39]), Diccionario de Autoridades (edición facsímil), Madrid, Gredos. 
Real Academia Española (1999), Ortografía de la lengua española, Madrid, Espasa-Calpe.

Rosales Solís, María Auxiliadora (2010), "El español de Nicaragua”, en M. A. Quesada Pacheco (coord.), El espanol hablado en América Central. Nivel fonético, Madrid / Fráncfort, Iberoamericana / Vervuert, pp. 137-154.

Sánchez-Prieto, Pedro y Ana Flores Ramírez (2005), Textos para la historia del español IV. Archivo Regional de la Comunidad de Madrid, Alcalá de Henares, Universidad de Alcalá.

\section{El texto editado}

\section{SEQUEHL}

AGN (México), Inquisición (61), vol. 854, p. 359.

s.f. [ca. 1736] (s.l. [León, León], Nicaragua)

Oración heterodoxa sobre la Pasión de Cristo (Oración del Santo Sepulcro).

Anónimo

Papel

Buen estado de conservación

José Luis Ramírez Luengo

\section{Transcripción PALEOgráficA}

$\{\mathrm{h} \operatorname{1r}\}\{1\}[\mathrm{cruz}]$

\{2\} Orazion Denues ttro Seño Jesu Christo Copia $\{3\}$ Copia de una Re Lazion, Que Fue allada en el Santo $\{4\}$ sepulCro LaCual tiene $\mathrm{SS}<\mathrm{u}>\mathrm{San}<$ tida $>$ en suoratorio Ynuestrore $\{5\}$ Rei y Seño en el Sullo La Cual Abiendo Santta Brigida \{6\} Ysanta Ysabel reina Deungria pusttose enora Sion $\{7\}$ Seles Aparesio Nuesttro seño y les digo HiJas mias $\{8\}$ Pues tanto deseas el Saber Los tormen tos $\mathrm{q}<\mathrm{ue}>$ Padisi $\{9\}$ enel dis Curso de mi pasion $\mathrm{p}<$ or $>$ re 
dimir al genero hu\{10\}mano es Como Sesigen Mis Pasos huno $\mathrm{p}<$ or $>$ huno

\{11\} Los sol dados Que me prendieron Fueron Siento y $\operatorname{Sin}\{12\}$ quenta \& Alabado sea mi dios y mi senor

$\{13\}$ La puñadas $\mathrm{q}<\mathrm{ue}>$ me dieron fueron tre Sientas \& $\{14\}$ alabado Sea my dios Y mi Seño \&

$\{15\}$ me dieron Siento Y dies Punta pies [margen: = alabadose $\{16\}$ asta en cas de anas $=$

$\{17\}$ Siento Y Seten ta Beses Me dieros En las Espaldas $\{18\}$ Para Le uan tar leme dela tiera Alabado Sea mi dios y mi señ

$\{19\}$ Fi ttirado, De labar ba Y Cabello tre Sientas Beses \& alaba $<$ do $>$

$\{20\}$ Mediero Sin Co mil Settesientos y settentta asotes [roto] $\{21\}$ Attado Ala coluna [roto: Y] Que daron en MiL Cuerpo myL $\{22\}$ eridas, Alabado Sea my dios Y mi Seño \&

$\{23\}$ Diero me de Aren Pugones Con La Cruz Y Cai quatro beses $\{24\}$ Alabado Sea mi dyos Y mi Señor \&

$\{25\}$ Lasangre que dera me Fueron Dies miL Seis Sientas $\{26\}$ Y Setenta Gotaz Alabado Sea Mi dios y mi seño

$\{27\}$ Las Perso nas q<ue $>$ de bota Mente En memoria $\{28\}$ des tos Paso de my Pazion ReSare Siete padre $\{29\}$ nuestros Y abe maria Con Su Gloria patri asta $\{30\}$ que Cun Pla el nu Mero de Go tas de Sang re que $\{31\}$ Llo dera Me en Mi passion $\{\mathrm{h} 1 \mathrm{v}\}$ $\{1\}$ Yque de Corazon Conten Ple Cada Paso des $\{2\}$ ta Re belasion que esta aqui Le prometo a $\{3\}$ Con Panar lo Yo y my querida Madre y mi ${ }^{\star}\{4\}$ Coros Angelicos a la ora de Sumuerte Y le prom ${ }^{\star * *}$ $\{5\}$ que Le sera Rebela do el dia de Su muerte

$\{6\}$ Sera Li bre de to dos Peli Gros que fue\{7\}ren en daño de Su Cuerpo a Siendo es ta $\{8\}$ ferboroza ora Sion An te my e figia Con $\{9\}$ re beren Sia Y que este engra Sia Mia le $\{10\}$ Prometo Librar lo de hor Ca de Sera ogado $\{11\}$ de morir Sin Con fision Lo librare de tra $\{12\}$ y Siones deRa llos $\mathrm{q}<\mathrm{ue}>$ es la Muerte Re Pen tina $\{13\}$ de oras Men Guadas La Muger q<ue $>$ estu $\{14\}$ Biere de Parto re Sando es taora Sion $\{15\}$ Como Sere fiere a la humilda Y Pa\{16\}Sien Sia Ydolores de la bir Gen Seño $\{17\} \mathrm{ra}$ Ym[interlineado: a]dre nues ta lea lludara Y SaCara $\{18\}$ del ries Go Amen Jesus Maria hi Jophe 
$\{19\}$ San[interlineado: to $]$ dios $=$ fuer te dios = Mi Seri Cor dioso dios $\{20\}$ aBe My Seri Cordia de mi Amen Jesus \&

$\{21\}$ Jesus $[c r u z]$ maria

$\{22\}$ Y Jophes $[\mathrm{cruz}] \mathrm{s}<$ an $>$ Guaquin

$\{23\}$ y Señor [cruz] Santaa $\{24\}$ na

\section{Presentación Crítica}

$\{$ h $1 r\}\{1\}[$ cruz]

$\{2\}$ Orazión de Nuestro Señó Jesú Cristo. Copia.

$\{3\}$ Copia de una relazión que fue allada en el Santo $\{4\}$ Sepulcro, la cual tiene su santidá en su oratorio y nuestro re $\{5\}$ rey y señó en el sullo; la cual, abiendo santa Brígida $\{6\}$ y santa Isabel, reina de Ungría, pústose en orasión, $\{7\}$ se les aparesió Nuestro Señó y les digo: "hijas mías, $\{8\}$ pues tanto deseás el saber los tormentos que padisí $\{9\}$ en el discurso de mi pasión por redimir al género hu\{10\}mano, es como se siguen mis pasos uno por uno:

\{11\} Los soldados que me prendieron fueron siento $y$ $\sin \{12\}$ cuenta; alabado sea mi Dios y mi Senor. $\{13\}$ La puñadas que me dieron fueron tresientas; $\{14\}$ alabado sea mi Dios y mi Señó. $\{15\}$ Me dieron siento y dies puntapiés $\{16\}$ asta en cas de Anás; alabado $\mathrm{se}^{23} \cdot\{17\}$ Siento y setenta beses me dieros en las espaldas $\{18\}$ para levantarme de la tiera; alabado sea mi Dios y mi Señ <or > . \{19\} Fi tirado de la barba y cabello tresientas beses; alabado. $\{20\}$ Me diero sinco mil setesientos y setenta asotes $\{21\}$ atado a la coluna, y quedaron en mi cuerpo mil $\{22\}$ eridas; alabado sea mi Dios y mi Señó. $\{23\}$ Diérome de arempugones con la cruz, y caí cuatro beses; $\{24\}$ alabado sea mi Dios y mi Señor. $\{25\}$ La sangre que deramé fueron dies mil seis sientas $\{26\}$ y setenta gótaz; alabado sea mi Dios y mi Señó.

\footnotetext{
${ }^{23}$ Se modifica la ubicación de este segmento respecto a su posición original en el texto para ofrecer una lectura más coherente del fragmento; tal modificación parece avalada no solo por el significado, sino también por la presencia del igual $<=>$, que actúa en la escritura dieciochesca como un signo de llamada de nota al margen dentro de un texto, similar al asterisco $<^{*}>$ actual (RAE, 1999: 87).
} 
\{27\} Las personas que debota mente, en memoria $\{28\}$ d'estos paso de mi pazión, resare siete padre $\{29\}$ nuestros y abemaría con su gloria patri asta $\{30\}$ que cumpla el número de gotas de sangre que $\{31\}$ llo deramé en mi passión $\{\mathrm{h} 1 \mathrm{v}\}\{1\}$ y que de corazón contemple cada paso d'es\{2\}ta rebelasión que está aquí, le prometo a $\{3\}$ companarlo, yo y mi querida madre y $\mathrm{mi}<\mathrm{s}>\{4\}$ coros angélicos, a la ora de su muerte, y le prom $<$ eto $>$ $\{5\}$ que le será rebelado el día de su muerte.

\{6\} Será libre de todos peligros que fue\{7\}ren en daño de su cuerpo asiendo esta $\{8\}$ ferboroza orasión ante mi efigia con $\{9\}$ reberensia y que esté en grasia mía; le $\{10\}$ prometo librar lo de horca, de ser aogado, $\{11\}$ de morir sin confisión, lo libraré de tra $\{12\}$ isiones, de rallos (que es la muerte repentina), $\{13\}$ de oras menguadas. La muger que estu\{14\}biere de parto, resando esta orasión, $\{15\}$ como se refiere a la humildá y pa $\{16\}$ siensia y dolores de la birgen, seño\{17\}ra y madre nuesta, le alludará y sacará $\{18\}$ del riesgo”. Amén, Jesús, María y Jofé. \{19\} Santo Dios, fuerte Dios, misericordioso Dios, $\{20\}$ abé misericordia de mí. Amén, Jesús.

$\{21\}$ Jesús [cruz] María

$\{22\}$ Y Jofés [cruz] san Guaquín

$\{23\}$ Y señor $[$ cruz] santa $A\{24\}$ na. 


\section{Facsímil}

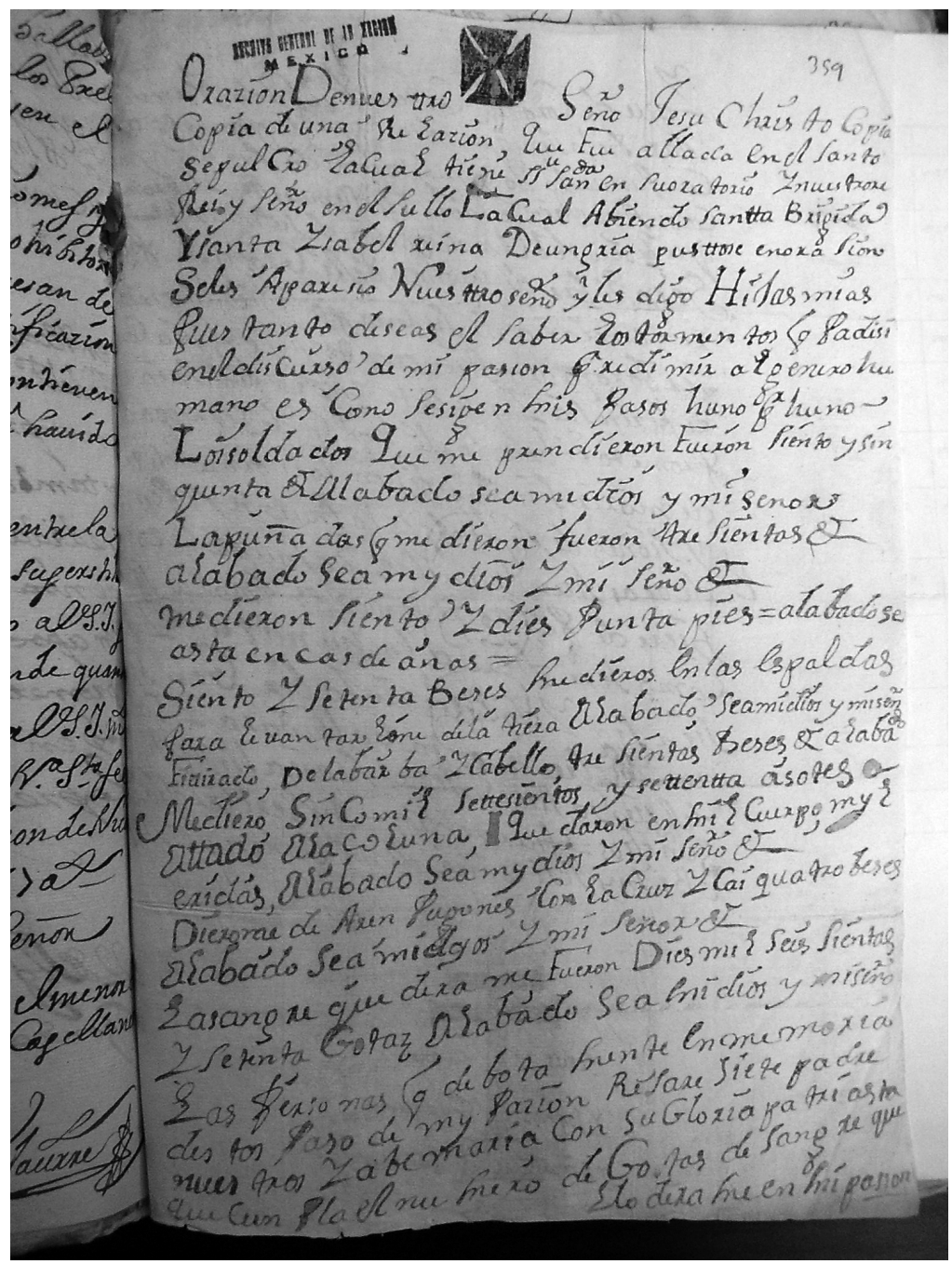

AGN (México), Inquisición (61), vol. 854, p. 359r. 
Manos inhábiles e historia del español de América: un ejemplo de la Nicaragua dieciochesca

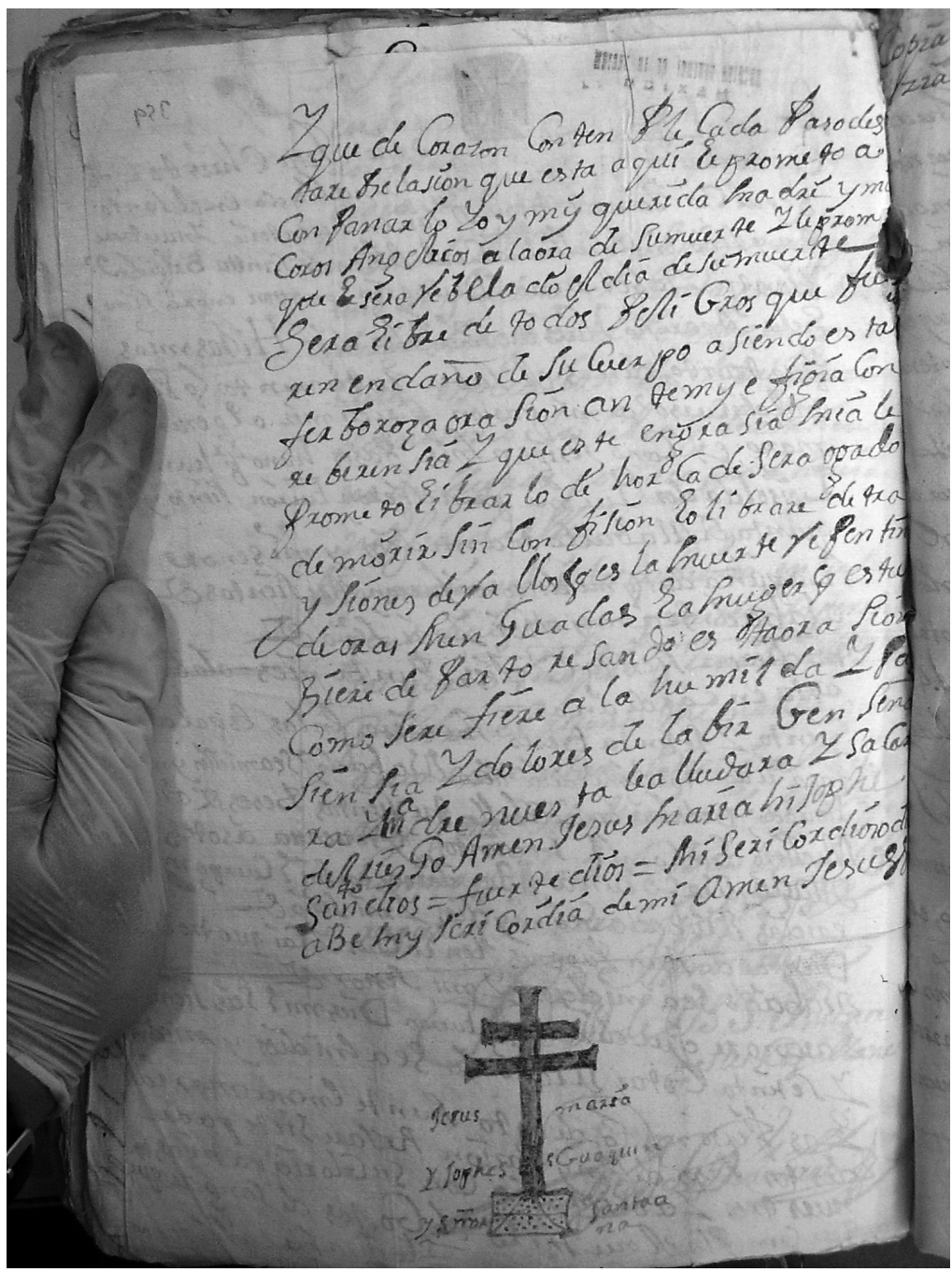

AGN (México), Inquisición (61), vol. 854, p. 359v. 